\title{
Awake Burr Hole Drainage Lessons Learned from 173 Experience of Local Anesthesia
}

\author{
Jin Mo Cho*
}

Department of Neurosurgery, Korea

ISSN: 2637-7748

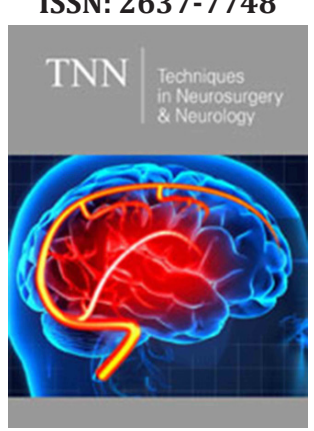

*Corresponding author: Jin Mo Cho, Department of Neurosurgery, Korea

Submission: 谐 October 08, 2020

Published: December 22, 2020

Volume 3 - Issue 5

How to cite this article: Jin Mo Cho. Awake Burr Hole Drainage Lessons Learned from 173 Experience of Local Anesthesia. Tech Neurosurg Neurol. 3(5). TNN. 000574. 2020

DOI: 10.31031/TNN.2020.03.000574

Copyright@ Jin Mo Cho, This article is distributed under the terms of the Creative Commons Attribution 4.0 International License, which permits unrestricted use and redistribution provided that the original author and source are credited.

\begin{abstract}
Background: Chronic subdural (CSDH) may happen after several day or week after mild to moderate head trauma, especially old age population. Usually, the procedure was done under general anesthesia, the procedure should be done by under local anesthesia due to several lesion. We routinely performed burr hole drainage under local anesthesia and report its benefits.
\end{abstract}

Methods: We retrospective review 173 patients with CSDH operated by burr hole under local anesthesia from March 2010 to February 2018.

Result: The age range was from 20 to 90 years with mean age of 66.5 years. There were $122(70.5 \%)$ males and $51(29.5 \%)$ females. There were 95 (54.9\%) of patients who had the right side, $65(37.6 \%)$ in the left side, and 25 (14.5\%) in bilateral CSDH. Radiologic follow-up showed removal of the CSDH in all cases with minimum residual in the early postoperative period and complete resolution at late follow-up. All patients were showed good clinical outcomes comparing preoperative period. post-op hospital stay day was 4.5 days (range 3-8). No patient required revision and no acute hematoma. There was no significant adverse event, during and after operation due to local anesthesia.

Conclusion: Chronic subdural hematoma could be removed by local anesthesia. The intraoperative pain could be well controlled by local anesthesia. We think that awake burr hole drainage under local anesthesia might be shortened hospital stay and prevent the complications of general endotracheal anesthesia, especially old or poor general condition patient.

\section{Introduction}

Chronic subdural hematoma (CSDH) is an encapsulated collection of old blood partially or completely liquefied and located between the dura and arachnoid mater1. Advanced age because atrophic brain associated with large subdural space predisposes the elderly people to the development of CSDH 14. Advanced age cause coexisting systemic disease usually caused a problem for general anesthesia in this old, aged patient group 6. Treatment of CSDH is usually surgical and frequently done by one of three procedures: twist drill craniostomy (opening less than $1 \mathrm{~cm}$ ), burr hole craniotomy, and craniotomy 13. In these procedures, burr hole craniostomy is the most used technique for evacuation of subdural hemorrhage. The aim of this study is to evaluate the outcome of burr hole under local anesthesia in the treatment of CSDH using absorbable skin suture [1-8].

\section{Method}

This study was approved by the Institutional Review Board of our hospital which waved the informed consent due to respective nature of study. We retrospective review 173 patients with CSDH operated by burr hole under local anesthesia from March 2010 to February 2018. Exclusion criteria included age below 19 years, multilocular hematomas. All the diagnosis was determined based on computed tomography (CT); CSDH was classified into hypodense hematomas, varying density hematomas and isodense or slightly hyperdense hematomas. Burr hole craniostomy was performed under local anesthesia in all patients. Local anesthesia was used in all cases with $10 \mathrm{ml}$ Lidocaine injected subperiosteally and subcutaneously. 
Scalp incision is $3 \mathrm{~cm}$ down to the periosteum then exposure of the skull. Single burr hole with $1 \mathrm{~cm}$ diameter was done, followed by dural cauterization, and then durotomy using no.11 knife. Drainage catheter $(10.5 \mathrm{Fr})$ was inserted in the subdural space and drain connected to a closed bag. Closure of the wound is after the hemostasis of the scalp edges using absorbable suture material. The patient was transferred directly to the ward in a flat position, and oral feeding is allowed directly. The drain amount was maximum 150cc per day. The patient was discharged after pulling out the drain catheter confirming CT f/u and there was no CSDH to drain. Scalp incision was explained in the outpatient clinic due to absorbable suture.

\section{Results}

Table 1: Clinical and radiologic presentation.

\begin{tabular}{|c|c|c|c|}
\hline \multirow{4}{*}{ Etiology } & Trauma & 140 & 80.9 \\
\cline { 2 - 4 } & Antiplatelet & 5 & 2.3 \\
\cline { 2 - 4 } & Coagulopathy & 3 & 1.7 \\
\cline { 2 - 4 } & Unknown & 25 & 14.5 \\
\hline \multirow{3}{*}{ Clinical } & Headache & 83 & 47.9 \\
\cline { 2 - 4 } & Hemiparesis & 141 & 81.5 \\
\cline { 2 - 4 } & Disturbed consciousness & 5 & 2.9 \\
\hline \multirow{3}{*}{ Sidedness } & Right & 85 & 49.1 \\
\cline { 2 - 4 } & Left & 65 & 37.6 \\
\cline { 2 - 4 } & Both & 23 & 13.3 \\
\hline
\end{tabular}

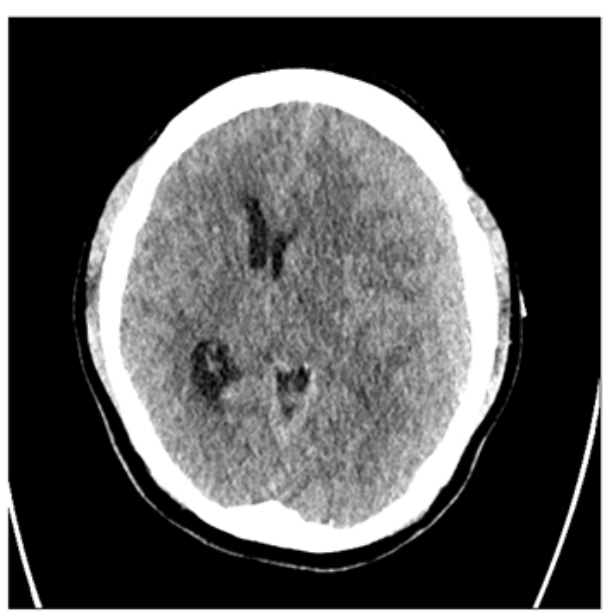

Figure 1: 84-year-old male patient presented with headache and right-side weakness. Brain CT showed a CSDH with midline shift.

The age range was from 20 to 90 years with mean age of 66.5 years (Table 1). There were 122 (70.5\%) males and 51 (29.5\%) females. Clinically, headache was found in 83 (47.9\%), hemiparesis in $141(81.5 \%)$. There were $85(49.1 \%)$ of patients who had the right side, $65(37.6 \%)$ in the left side, and 23 (13.3\%) in bilateral CSDH (Table 1). Radiologic follow-up showed removal of the CSDH in all cases with minimum residual in the early postoperative period and complete resolution at late follow-up. All patients were showed good clinical outcomes comparing preoperative period. Mean post-op hospital stay day was 4.5 days (range 3-8).
19 patients $(10.7 \%)$ show residual CSDH cannot be drained but follow up study shows complete resolution of the CSDH. No patient required revision and no acute hematoma formation. Illustrative case (residual hematoma) A 84-year-old male patient presented with headache and right-side weakness. Brain CT showed a CSDH with midline shift. We performed burr hole drainage under local anesthesia. Postoperative patient response was good, and the patient's symptoms was gone. However, f/up CT showed residual CSDH [9-14]. The patient symptom was good, so we wait and f/up the patient. The patient symptom was consistently good, so we did not perform further treatment and after 6 months f/up CT showed total resolution of residual CSDH (Figures 1-3).

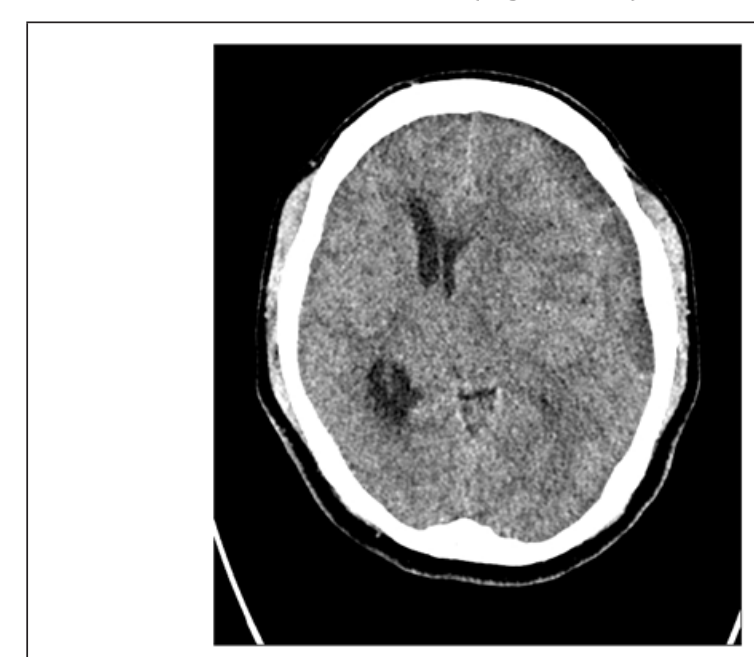

Figure 2: Postoperative follow up CT showed residual CSDH.

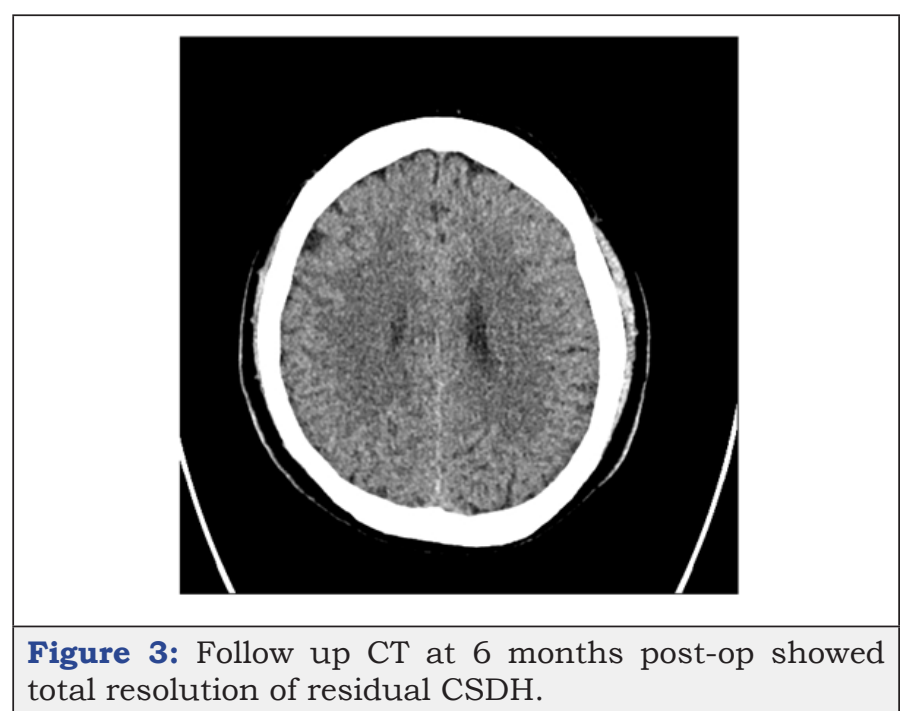

\section{Discussion}

$\mathrm{CSDH}$ is a common neurosurgical problem in old age. Burr hole craniostomy is considered the gold standard for surgical treatment of CSDH3, 12 We performed surgery with minimal anesthetic agents and because minimal complication caused by general anesthesia. Generally, for patients who have coexisting complex systemic disease or old aged, local anesthesia is a more favored method during surgery. General anesthesia can cause 
systemic illness aggravation especially old, aged patients. Local anesthesia was used by many other authors. The surgical treatment for subdural hematoma varies from twist drill craniostomy to large craniotomy procedures. The mean operative time was $25 \mathrm{~min}$ in our study, this time is equal to that of gospel $25.5 \mathrm{~min}$ with a range from 15 to $45 \mathrm{~min}$ ), and this is another advantage or our technique. We cannot find recurrence in our study, and this is one of the best results reported in chronic subdural hematoma surgery; the rate of recurrence after burr hole evacuation varies between 2.7 and $34 \%$ but our result shows no recurrence $2,7,9-11$. The acute subdural hematoma formation was none. This is due to the small single scalp incision and control scalp bleeding easily. We performed surgery using absorbable skin suture, so hospital stay was short. Absorbable skin suture material resolves 3 weeks spontaneously, so there is no need to wait for suture material removal. Residual hematoma was common presentation. Burr hole drainage was removal of chronicacute hemorrhage pressure effect. Residual hemorrhage can be resolution spontaneously but If patients' condition is good "wait and see" policy was considered. If patient symptom was consistent it may need re-operation, but our study did not show the case. Limitation of this study was minimal sample size and no result for pain score. The average numeric pain score was 2.3 (rage $0-6$ ), but all the patient was not inspected. However, there was no patient for altering general anesthesia for the pain. We need follow up study for the pain score.

\section{Conclusion}

Treatment of CSDH by burr hole using local anesthesia is safe and effective for especially for old CSDH patients with short hospital stay, low cost, and lower complication rate.

\section{References}

1. Agrawal A (2010) Bilateral biconvex frontal chronic subdural hematoma mimicking extradural hematoma. J Surg Tech Case Rep 2(2): 90-91.

2. Aung T, Wong W, Mo H, Tsang C (1999) Management of chronic subdural haematoma: burr hole drainage, replacement with hartmann's solution, and closed-system drainage. Hong Kong Med J 5(4): 383-386.
3. Drapkin AJ (1991) Chronic subdural hematoma: pathophysiological basis for treatment. Br Neurosurg 5(5): 467-473.

4. Ernestus RI, Beldzinski P, Lanfermann H, Klug N (1997) Chronic subdural hematoma: surgical treatment and outcome in 104 patients. Surg Neurol 48(3): 220-225.

5. Erol FS, Topsakal C, Ozveren MF, Kaplan M, Tiftikci MT (2005) Irrigation vs. closed drainage in the treatment of chronic subdural hematoma. J Clin Neurosci 12(3): 261-263.

6. Guzel A, Kaya S, Ozkan U, Aluclu MU, Ceviz A, et al. (2008) Surgical treatment of chronic subdural haematoma under monitored anaesthesia care. Swiss Med Wkly 138(27-28): 398-403.

7. Khadka N, Sharma G, Roka Y, Kumar P, Bista P, et al. (2008) Single burr hole drainage for chronic subdural haematoma. Nepal Med Coll J 10(4): 254-257.

8. Lee JY, Ebel H, Ernestus RI, Klug N (2004) Various surgical treatments of chronic subdural hematoma and outcome in 172 patients: is membranectomy necessary? Surg Neurol 61(6): 523-527.

9. Markwalder TM, Steinsiepe KF, Rohner M, Reichenbach W, Markwalder H (1981) The course of chronic subdural hematomas after burr-hole craniostomy and closed-system drainage. J Neurosurg 55(3): 390-396.

10. Mori K, Maeda M (2001) Surgical treatment of chronic subdural hematoma in 500 consecutive cases: clinical characteristics, surgical outcome, complications, and recurrence rate. Neurol Med Chir 41(8): 371-381.

11. Tabaddor K, Shulman K (1977) Definitive treatment of chronic subdural hematoma by twist-drill craniostomy and closed-system drainage. J Neurosurg 46(2): 220-226.

12. Wakai S, Hashimoto K, Watanabe N, Inoh S, Ochiai C, et al. (1990) Efficacy of closed-system drainage in treating chronic subdural hematoma: a prospective comparative study. Neurosurgery 26(5): 771-773.

13. Weigel R, Schmiedek P, Krauss J (2003) Outcome of contemporary surgery for chronic subdural haematoma: evidence based review. J Neurol Neurosurg Psych 74(7): 937-943.

14. Yang AI, Balser DS, Mikheev A, Offen S, Huang JH, et al. (2012) Cerebral atrophy is associated with development of chronic subdural haematoma. Brain Inj 26(13-14): 1731-1736. 